\title{
SPH Simulations of the Chemical and Dynamical Evolution of the
}

\author{
Galactic Bulge \\ T. TSUJIMOTO, K. NOMOTO, T. SHIGEYAMA and Y. ISHIMARU \\ Department of Astronomy, University of Tokyo, Bunkyo-ku, Tokyo 113, Japan
}

\begin{abstract}
We simulate the chemical and dynamical evolution of the galactic bulge with the smoothed particle hydrodynamics (SPH) method. We calculate the early phase of galaxy formation in which the bulge is formed through a burst of star formation. The calculated abundance distribution function of stars in the bulge is consistent with the observations of bulge $\mathrm{K}$ giants, if the heavy element yields are three times larger than those expected from Salpeter's IMF.
\end{abstract}

Key words: galaxies: dynamics - abundances - supernovae

The observed abundance distribution function of bulge K giants (Rich 1990) shows the presence of stars with a wide range of metallicity $(-1 \leq[\mathrm{Fe} / \mathrm{H}] \leq+1)$. Matteucci \& Brocato (1990) constructed one zone chemical evolution models for the bulge and found that the observed distribution function is reproduced only if the timescale of galaxy collapse is as short as $10^{7} \mathrm{yr}$. This seems to be too short to be compatible with the free fall timescale, thus indicating the necessity of realistic hydrodynamical simulations. We construct three dimensional models for the chemical and dynamical evolution with SPH, and calculate the collapse of a protogalaxy until a burst of star formation ceases.

Our model has three components, i.e., gas, dark matter and stars, with the Jeans criterion of star formation. During collapse, the thermal and chemical evolutions of gases and stars are calculated by taking into account radiative cooling and supernova heating as well as enrichment of heavy elements due to supernova explosions.

The results are as follows. The core which consists of stars and gas are formed in the central part of dark matter at $\sim 2 \times 10^{8} \mathrm{yr}$ (Fig.1). A burst of star formation occurs in a dense gaseous core where radiative cooling is very effective. Massive stars then explode as type II supernovae and rapidly contaminate the core with heavy elements (Fig.2). The metallicity $[\mathrm{Fe} / \mathrm{H}]$ at the peak of the abundance distribution function of stars in the core is lower than that of bulge $\mathrm{K}$ giants, if the yield expected from Salpeter's IMF is used. With the three times lager yield, the average metallicity of gas in the core becomes about twice the solar abundance. Some stars are formed from gases of more metal rich than the average due to the inhomogeneous metal contamination. To reproduce the abundance distribution function of bulge $\mathrm{K}$ giants as seen in Fig.3, it is very important to consider the inhomogenity which does not appear in one zone models. In addition, our model predicts that the heavy elements in the bulge stars are mostly produced from type II supernovae. This is consistent with the observations in which the abundance ratios between the $\alpha$-elements and $\mathrm{Fe}$ in the bulge $\mathrm{M}$ giants are significantly higher than the solar ratios (Rich 1992).

\section{References}

Matteucci F., Brocato E., 1990, $A p J, 365,539$.

Rich R.M., 1990, ApJ, 362, 606.

Rich R.M., 1992, in IAU Symposium 149, The Stellar Populations of Galaxies (Kluwer), p. 29. 

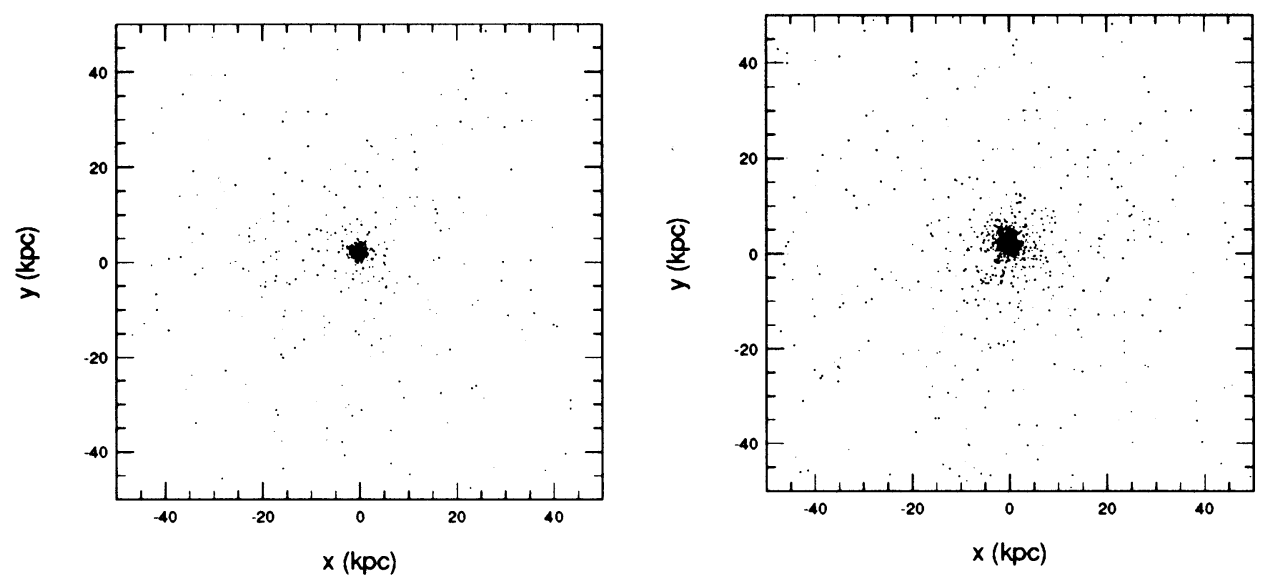

Fig. 1. Distributions of gas (left) and dark matter (right) at $2.3 \times 10^{8} \mathrm{yr}$.
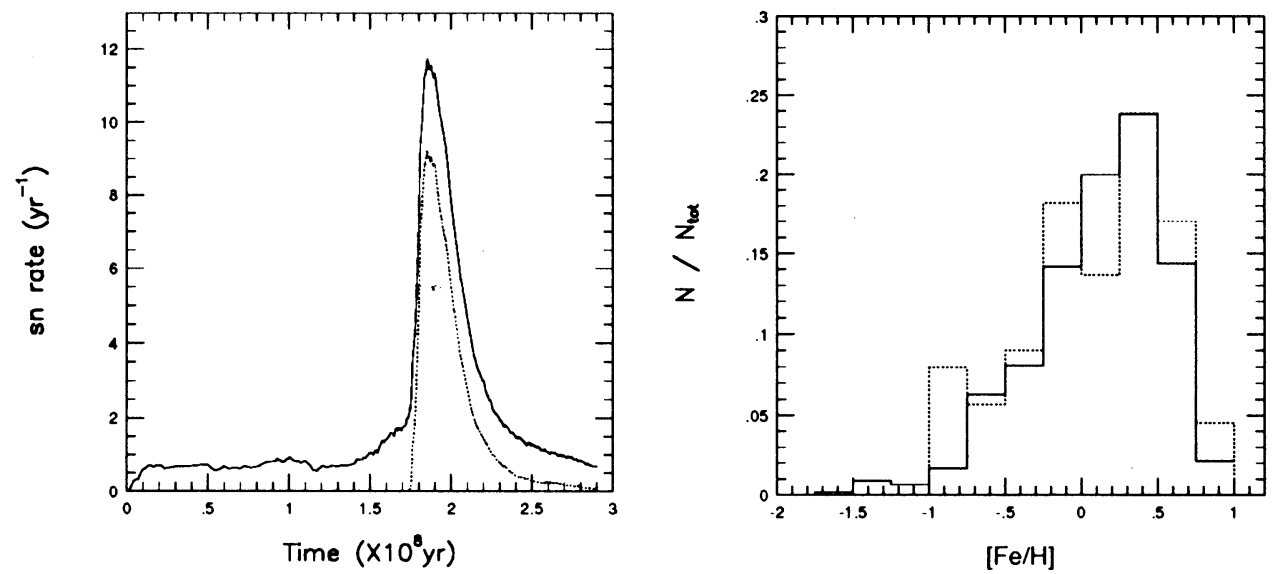

Fig. 2. Supernovae rate vs. time. The solid and dotted lines show the total supernova rate, and the rate in the core within $2 \mathrm{kpc}$, respectively (left).

Fig. 3. Abundance distribution function of stars in the core (solid) as compared with that of bulge K giants (dotted:Rich 1990) (right). 